\title{
Inflation expectations and macroeconomic dynamics: the case of rational versus extrapolative expectations
}

\author{
Marji Lines ${ }^{\mathrm{a}, *}$ Frank Westerhoff ${ }^{\mathrm{b}}$ \\ ${ }^{a}$ Department of Statistics, University of Udine, Via Treppo 18, 33100 Udine, Italy \\ ${ }^{\mathrm{b}}$ Department of Economics, University of Bamberg, Feldkirchenstrasse 21, 96045 \\ Bamberg, Germany
}

\begin{abstract}
The motivation of this paper is to understand the effects of coupling a macroeconomic model of inflation rate dynamics, relying on an aggregate expectation, to a heterogeneous expectations framework. A standard macroeconomic textbook model, consisting of Okun's law, an expectations-augmented Phillips curve and an aggregate demand relation, is extended to allow agents the possibility of selecting between different types of forecasting strategies to predict the future inflation rate. Rules that have produced low prediction errors in the recent past are favored by agents. Here we consider trend-following and rational expectations. Using a mixture of analytical and numerical tools we investigate the model's dynamics and discuss the conditions under which the extended model leads to endogenous fluctuations in macroeconomic variables. Some preliminary results are offered for the case in which a Taylor-like monetary policy rule is included in the model.

JEL classification: C61; C62; E31; E32

Keywords: Heterogeneous expectations; expectation formation; dynamic macroeconomics; Okun's law and Phillips curve; nonlinearities and chaos; bifurcations; intermittency
\end{abstract}




\section{Introduction}

The aim of our paper is to explore the interplay between heterogeneous expectation formation and macroeconomic dynamics. Our starting point is the monetary model. Its basic ingredients are Okun's law and the expectations-augmented Phillips curve, used to describe the supply side of the economy, and an output growth relation driven by changes in both nominal money growth and inflation. The novel feature of our model is how we treat the agents' expectation formation behavior.

To make matters as simple as possible, agents either use a sophisticated, costly predictor or they use an easy, cheap predictor (as in Brock and Hommes 1997). Agents select between competing forecasting strategies based on forecasting accuracy, measured in terms of squared forecasting errors. The aggregate expectation, a weighted average of the two, is a factor in the expectations-augmented Phillipscurve. Except for the interacting expectation formation process, we therefore use a standard (textbook) macroeconomic model (Blanchard, 2005).

We have investigated the effects of heterogeneous expectations on the dynamics of the model through a combination of mathematical analysis and numerical simulations. The results should prove useful to the increasing number of scholars who are integrating the heterogeneous expectation framework into their own modeling

\footnotetext{
* Corresponding author. Tel: +39 0432249582; fax: +39 0432249595.
}

The authors thank three anonymous referees, the editor (Cars Hommes) and Alfredo Medio for helpful comments and suggestions. M. Lines thanks the Italian Ministry of Universities, the Universities of Udine and Venice, and the Friuli-Venezia Giulia Region for grants to develop the iDMC software.

Email address: lines@dss . uniud. it (Marji Lines). 
environment ${ }^{1}$. Related studies include Westerhoff (2006a), Lines and Westerhoff (2006a) and Lines (2007a). In these papers, a basic goods market model is used and agents switch between prediction strategies depending on how far the economy has deviated from its long-run equilibrium value. For instance, there is a shift from extrapolative expectations to reversion expectations as national income moves away from its equilibrium value. Westerhoff (2006b) also uses a simple goods market model but focuses on a situation in which predictor choice depends on an evolutionary fitness measure.

Branch and McGough $(2006,2008)$ introduce heterogeneous expectation formation in a New Keynesian framework. In their model, agents have to predict both the future inflation rate and the future output level. A key goal of their papers is to derive a setting in which the heterogeneous expectation formation is consistent with a utility maximization framework. Another interesting contribution is by Franke (2007) in which agents use different forecasting rules to predict the inflation rate. Then the average of these inflation forecasts is used as a proxy for the current inflation climate which, in turn, is relevant for the agents' price and wage setting behavior, modeled via the Phillips curve. Anufriev et al. (2008) and de Grauwe (2008) are two recent interesting examples in which monetary policy rules are investigated in macroeconomic models with heterogeneous expectations.

Closely related to these contributions are models in which agents may display some kind of learning behavior (see Evans and Honkapohja 2001 for a general survey on learning in macroeconomics). For instance, Berardi (2007) considers a model which is populated by two different types of agents who learn through recursive $\overline{1}$ The most obvious applications have been to the modeling of financial markets, such as in the works of Day and Huang (1990), Lux (1995), Brock and Hommes (1998), Chiarella, Dieci and Gardini (2002). 
least squares techniques the parameter values of their forecasting strategies. Tuinstra and Wagener (2006) go one step further and study an evolutionary competition between two different estimation procedures. Overall, these models find that bounded fluctuations in macroeconomic variables may emerge even in the presence of learning behavior. Moreover, Marcet and Nicolini (2003) show that their model of "quasi-rational learning" mimics some key stylized facts observed during the recurrent hyperinflations experienced by several countries in the 1980s quite well. Other interesting learning models include Branch and Evans (2006) and Honkapohja and Mitra (2006). Given the explanatory power of these approaches they appear quite relevant for policy evaluation.

The complicated dynamics of our models are obviously due to the nonlinearities in the expectation framework. There are, of course, a number of other interesting macroeconomic models which use other mechanism to generate endogenous dynamics, e.g., Day (1999), Rosser (2000), Lines (2005), Puu and Sushko (2006). In our case, fluctuating long run dynamics are due to a permanent evolutionary competition between the prediction rules. For example, if agents have the choice between rational and extrapolative expectations, the dynamics of the model may be sketched as follows. Suppose that a large fraction of the agents rely on the rational predictor. Then the dynamics is stable and a convergence towards a "normal" steady state sets in. However, the system does not necessarily settle down on this fixed point. For instance, close to the steady state the forecasting accuracies of both predictors become similar. If a sufficient number of agents switch to the extrapolation predictor, the steady state becomes unstable and oscillation in key macroeconomic variables are triggered. Rational expectations may gain in popularity again when the prediction errors of extrapolative expectations become strong. This sequence repeats itself, with the dynamics further complicated by macroeconomic feedback 
processes (which will be explained below).

In Section 2 we present the full model. In Section 3 we investigate dynamics in the case of rational vs. extrapolative predictors. Conclusions are offered in Section 4. In the Mathematical Appendix we derive the critical values of parameters for loss of local stability of the unique fixed point for both pairs of expectations.

\section{The model}

The basic model we use to describe inflation rate dynamics combines three macroeconomic relations: Okun's law, an expectations-augmented Phillips curve and an aggregate demand relation. These quite standard macroeconomic tools are discussed in detail in Blanchard (2005), for example. The novel feature of our model is the introduction of a framework for exploring the effects of various expectation operators on the dynamics of the inflation rate. We first derive the results of assuming a single expectation strategy. Then, following Brock and Hommes (1997, 1998), we assume that the agents have the choice between different types of forecasting rules and select between them depending on their predictive capacity, which plays the role of an evolutionary fitness measure.

\subsection{The macroeconomy}

Consider Okun's law, which states that changes in unemployment are related to output growth. We denote by $u_{t}$ the unemployment rate, by $g_{t}$ the output growth rate and by $g_{n}$ the so-called normal output growth rate, and Okun's law may be formalized as

$$
u_{t}-u_{t-1}=-\beta\left(g_{t}-g_{n}\right), \quad \beta>0 .
$$


Accordingly, output growth above (below) normal leads to a decrease (increase) in the unemployment rate. To maintain a stable unemployment rate, output growth obviously must be equal to the normal output growth. Recall that the empirical support for Okun's law is very solid. Estimates of the normal output growth rate are generally around 3 percent for the U.S. economy.

There are currently several versions of the Phillips curve. Here we make use of the expectations-augmented Phillips curve in which inflation is conditional on expected inflation and the deviation of the unemployment rate from its natural rate. Hence,

$$
\pi_{t}=\pi_{t}^{e}-\alpha\left(u_{t}-u_{n}\right), \quad \alpha>0,
$$

where $u_{n}$ is the normal rate of unemployment. The time index of $\pi_{t}^{e}$, the aggregate expected inflation rate, refers to the period for which the expectation is formed. The agents' information set includes the past values of relevant variables up to time period $t-1$.

The economic motivation of (2) is as follows. If agents expect a higher inflation, then workers demand (and obtain) higher wages. Since the firms use a mark up pricing rule, they consequently increase prices and so the inflation rate increases. In addition, if the unemployment rate decreases, workers have a stronger bargaining power and are able to negotiate higher wages. Again, firms do mark up pricing and the inflation rate rises.

The aggregate demand is defined by a simple, linear relation in which output growth adjusts to the difference between nominal money growth and the inflation rate

$$
g_{t}=m-\pi_{t}
$$

The nominal money growth rate $m$ is constant over time. Recall that (3) is consis- 
tent with (i.e. may be derived from) the basic IS-LM framework. For instance, an increase in the real money stock leads to a decrease in the interest rate. As a result, the demand for goods and thus also output increases.

From (1)-(3) we can derive

$$
\pi_{t}=\frac{\alpha \beta\left(m-g_{n}\right)}{1+\alpha \beta}+\frac{\pi_{t-1}}{1+\alpha \beta}+\frac{\pi_{t}^{e}-\pi_{t-1}^{e}}{1+\alpha \beta}
$$

i.e. the inflation rate in period $t$ depends on the inflation rate in period $t-1$ and on the expected inflation rates for periods $t$ and $t-1^{2}$.

The equilibrium inflation rate value, $\bar{\pi}$, in terms of expectations is

$$
\bar{\pi}=\left(m-g_{n}\right)+\alpha \beta\left(\pi_{t}^{e}-\pi_{t-1}^{e}\right) .
$$

Once there are no further changes in expectations we have

$$
\bar{\pi}=m-g_{n}=\pi_{n}
$$

that is, the equilibrium inflation rate is given by the distance between money and output growth. The particular forms of strategies used in the following always imply that agents commit no forecasting errors in equilibrium (in equilibrium all agents predict $\pi_{n}$ ). If expectations are eliminated, the model (4) is characterized by monotonic convergence to equilibrium. Otherwise, the stability of $\pi_{n}$, and the existence and types of other limit sets, depend on the expectations set-up as we discuss in what follows.

\footnotetext{
$\overline{2}$ To see this solve (2) for $u_{t}$. Doing the same for $u_{t-1}$, we can calculate the difference $u_{t}-u_{t-1}$ and substitute back into (1). Solving this expression for $\pi_{t}$ and making use of (3) gives (4).
} 


\subsection{Expectation formation}

We first investigate the dynamics of the inflation rate equation (4) with single strategies, all of which are linear. Then the coupling of homogeneous expectations to the macro model through (4) results in linear models whose dynamics are readily determined.

In principle, agents may make efficient use of the available information and form rational expectations,

$$
\pi_{t}^{R}=\pi_{t}
$$

and the aggregate expectation is always realized. Model (4) then assigns $\pi_{t}=\pi_{n}$ for all $t$, because the inflation rate is perfectly predicted and the inflation rate never deviates from its normal rate. One may argue that the rational expectations operator has a super-stabilizing effect on the inflation rate equilibrium.

Agents may also choose to base their expectations on a much smaller part of the information. Here we consider that agents may decide to follow the trend of the very recent past, an inefficient but simple, and one could argue, common practice. If all agents are extrapolators the aggregate expectation is

$$
\pi_{t}^{E}=\pi_{t-1}+\gamma\left(\pi_{t-1}-\pi_{t-2}\right)
$$

Agents include a term with the direction of change of the inflation rate, with $\gamma$ indicating how strong the agents extrapolate past inflation trends into the future. This behavioral parameter indicates the agents' level of optimism/pessimism, and plays an important role in the stability of the equilibrium rate. If $\gamma$ is low the equilibrium is stable, but the normal inflation rate loses stability at $\gamma_{c}=0.5(1+\alpha \beta)$ as the modulus of a complex, conjugate pair of eigenvalues is one. For $\gamma>\gamma_{c}, \pi_{n}$ is no longer an attractor. The trend-following strategy is thus potentially destabilizing. 


\subsection{Empirical support}

In this section we present empirical evidence that views about the future inflation rate differ significantly among agents. For instance, analyzing survey data on inflation expectations, Mankiw et al. (2003) find that the interquartile range of inflation expectations for 2003 among economists ranges from 1.5 to 2.5 percent and that among the general public, the interquartile range of expected inflation ranges between 0 and 5 percent. Also Carroll (2003) and Pesaran and Weale (2006) report similar evidence and conclude that inflation expectations are not always formed in a fully rational way. A central issue then is how agents form inflation expectations in real economies and how we should model this. There exists a good deal of empirical research to guide us in this effort, we mention some of the most relevant below.

Experiments conducted by Simon (1955), Kahneman, Slovic and Tversky (1986) and Smith (1991) suggest that agents should be regarded as boundedly rational. Although people lack the cognitive capabilities to derive fully optimal actions, they should not be regarded as irrational. In fact, people strive to do the right thing. It may in many situations be more accurate to describe their behavior as rule-governed behavior. This means that people rely on a limited number of heuristic principles which have proven to be useful in the past.

When it comes to the formation of expectations, Heemeijer et al. (2009) find that agents tend to use simple linear forecasting rules based on recent information to form predictions. In particular, agents use extrapolative expectation formation rules which are common in so-called positive expectations feedback systems. Moreover, in such contexts these expectations may become self-confirming. One should note 
that in macroeconomic models, such as the one we develop in this paper, a positive expectations feedback may be inherent. For instance, if the majority of agents expects the inflation rate to increase, then, according to the expectations-augmented Phillips curve, it will increase (everything else being equal). Further support on the use of extrapolative expectations in the case of positive feedback systems is provided by Hommes et al. (2005) and Hommes et al. (2008).

In another interesting empirical work, Branch (2004) studies which forecast rules agents apply to predict the inflation rate. From survey data on inflation expectations, he concludes that agents rely on rules such as VAR forecasts, adaptive expectations or naive expectations. The VAR predictor, which takes past macroeconomic realizations such as the inflation rate, the unemployment rate, the interest rate or the money growth rate into account, is interpreted by Branch as a boundedly rational predictor "in the spirit" of rational expectations. Moreover - and this is quite relevant for our paper - he also finds that agents do not blindly follow one of these rules but dynamically select predictor functions. The proportion of agents that use each predictor varies inversely with the predictors mean squared prediction error. Hence, agents display some kind of learning behavior in the sense that they tend to select prediction rules that did well in the past. Depending on the setting, agents may switch quite quickly between strategies. Branch also finds evidence for a predisposition effect. Agents switch between predictors as the forecast accuracy warrants, however, there is inertia to these switches. This is because forecast errors must pass some threshold before agents abandon their previously selected predictor. In his setting, there is a predisposition for the more sophisticated VAR method. Further evidence for dynamic predictor selection and heterogeneous expectation formation in different environments is provided by Baak (1999), Chavas (2000), Westerhoff and Reitz (2003), Alfarano et al. (2005), Boswijk et al. (2007) and Branch (2007). 


\subsection{Heterogeneous expectation framework}

Recall that aggregate inflation expectations enter the macroeconomic model via the expectations-augmented Phillips curve. In the case of co-existing strategies, the aggregate expectation for the economy is a weighted average over the expectation for each type of strategy. Note that we include heterogeneous expectations in form of a weighted average of individual expectations into a macroeconomic model which has no explicit microfoundation. However, Anufriev et al. (2008) argue that for a linear macroeconomic model such as theirs, this is the most natural way to proceed. Also Lines and Westerhoff (2006a) and De Grauwe (2008), among others, follow this approach.

Our focus is on macroeconomies in which a stabilizing strategy (rational expectations) and a potentially destabilizing operator (extrapolative expectations) co-exist. The rational expectations predictor requires an information set substantially richer than that required by the extrapolative predictor and we assume that there is an associated cost. However, the cost parameter may include not only the actual cost of the strategy, in terms of collecting information, calculating, etc. but also a predisposition effect, in terms of reluctance to adopt a more difficult algorithm (see, for example, Branch 2004).

Then, with rational expectations $(\mathrm{R})$ against extrapolative trend-following $(\mathrm{E})$, the aggregate inflation rate is a weighted linear combination of the two:

$$
\pi_{t}^{e}=w_{t}^{E} \pi_{t}^{E}+w_{t}^{R} \pi_{t}^{R}
$$

where the relative weights of extrapolative and rational are denoted by $w_{t}^{E}$ and $w_{t}^{R}$, respectively. Agents are not constrained to a certain rule and compare their relative prediction performance. We assume that the agents prefer tools with a high fore- 
casting accuracy and rely on squared prediction errors as a (publicly observable) fitness measure. The attractiveness of the strategies are defined as follows

$$
a_{t}^{E}=-\left(\pi_{t-1}^{E}-\pi_{t-1}\right)^{2}, \quad a_{t}^{R}=-\left(\pi_{t-1}^{R}-\pi_{t-1}\right)^{2}-\kappa, \quad \kappa \geq 0
$$

where $\kappa$ represents the costs of forming rational expectations.

As in Brock and Hommes $(1997,1998)$, we update the fractions of agents using one or the other predictor via a discrete-choice model (Manski and McFadden, 1981). The weight of the extrapolative predictor is

$$
w_{t}^{E}=\frac{\exp \left(\lambda a_{t}^{E}\right)}{\exp \left(\lambda a_{t}^{E}\right)+\exp \left(\lambda a_{t}^{R}\right)}, \quad \text { or } \quad w_{t}^{E}=\frac{1}{1+\exp \left[\lambda\left(a_{t}^{R}-a_{t}^{E}\right)\right]}
$$

and $w_{t}^{R}=1-w_{t}^{E}$. The parameter $\lambda \geq 0$ measures how sensitive agents are to selecting the most attractive predictor. For instance, if $\lambda=0$, agents are unable to discriminate between the two predictors so that $w_{t}^{1}=w_{t}^{2}=0.5$. On the other hand, in the limit for $\lambda \rightarrow+\infty$, all agents select the best performing predictor.

\section{Rational versus extrapolative expectations}

We are now ready to explore interactions between the sophisticated rational expectations (or perfect foresight) predictor and the extrapolative trend-following predictor. We start with a study of the local dynamics using analytical methods, and then turn to numerical simulations for a deeper understanding of global dynamics and the motion on non-point attractors. Finally, we investigate the impact of a Taylor-like monetary policy rule on the dynamics of our model. 


\subsection{Analytical results}

Coupling the heterogenous expectation framework (7)-(11) with (4) we have:

$$
\pi_{t}=f\left(\pi_{t-1}, \pi_{t-2}, \pi_{t-3}, \pi_{t-4}\right),
$$

giving a fourth-order nonlinear law of motion, with a unique equilibrium inflation rate $\pi_{n}=m-g_{n}$. The time evolution of the unemployment rate and output growth may be expressed, respectively, as $u_{t}=h\left(\pi_{t}, \pi_{t-1}, \pi_{t-2}\right), g_{t}=k\left(\pi_{t}\right)$. Once the path of the inflation rate is determined, the evolution of the other two variables are determined and the steady state values of the unemployment rate and the output growth rate are equal to their normal values.

Analysis based on the Jacobian of the first-order system in current and lagged values of $\pi$, confirms a unique loss of local stability of $\pi_{n}$.

Proposition 3.1 The inflation rate equation (4) with heterogeneous expectations framework (7)-(11), extrapolative versus rational expectations predictors, has a unique fixed point $\pi_{n}$, which loses stability through a Neimark-Sacker (NS) bifurcation at critical value

$$
\gamma_{c}=\frac{\alpha \beta+\mu}{2 \mu}, \quad \mu=\frac{1}{1+e^{-\lambda \kappa}}
$$

(see Mathematical Appendix).

Note that Proposition 3.1 implies that if either information costs $(\kappa)$ or intensity of choice $(\lambda)$ are very high, the critical value for the extrapolation reaction parameter approaches $\gamma_{c}=0.5(1+\alpha \beta)$. On the other hand, if either $(\kappa)$ or $(\lambda)$ are near 0 , the critical value is larger, approaching $\gamma_{c}=0.5+\alpha \beta$. Then the equilibrium inflation rate $\pi_{n}$ is stable for all reaction parameter values $\gamma<0.5(1+\alpha \beta)$ and unstable for 
$\gamma>0.5+\alpha \beta$

Moreover, extensive numerical studies of the model suggest the following. The local NS bifurcation is subcritical, so that in a (sufficiently) small left neighborhood of $\gamma_{c}$, the curves bifurcating from (and enclosing) $\pi_{n}$ are repelling. This implies the "corridor stability" of $\pi_{n}$, that is, the basin of attraction of the equilibrium decreases until, at the critical parameter value, it disappears. Yet, the global dynamical behavior over much of the relevant parameter space is motion on, or close to, stable invariant curves. There is also a part of the parameter space characterized by the coexistence of these limit sets and the stable $\pi_{n}$. These properties suggest that the subcritical NS is accompanied by a two-parameter bifurcation known as a Chenciner (or crater) bifurcation (see Agliari 2006, Agliari et al. 2006, Gaunersdorfer et al. 2008 for economic applications and references). We turn to a study these dynamical aspects through numerical and graphical methods.

\subsection{Parameter sensitivity}

There are many parameters and we proceed by fixing a basic set of parameter values. For the parameters belonging to the macroeconomic textbook part of the model we set $g_{n}=0.02, u_{n}=0.05, m=0.05, \alpha=1, \beta=0.35$. The normal output growth rate is 2 percent, the normal rate of unemployment is 5 percent and the nominal money growth is 5 percent. Note that the slope parameters of the Phillips curve and Okun's law are also in line with empirical observations (Blanchard, 2005).

The heterogeneous expectations framework introduces a behavioral parameter $\lambda$, implying the propensity to switch between predictors, and a cost $\kappa$ for the sophisticated predictor which is also in part behavioral. For the heterogeneous expectations 
framework we typically set $\kappa=0.0001$, so that the actual and psychological costs of using rational expectations have the same weight as a 1 percent error in the attractiveness function (10).

The sensitivity parameter in the weight function (11) is set at $\lambda=12500$. To test the effect of this value on switching, we compare proportions of agents selecting one or the other predictor in given situations. Suppose that the prediction error of a strategy $\mathrm{A}$ is $1 \%$ less than the prediction error of a strategy B and that strategy B makes an error of 0.01 . Moreover, let $\kappa=0$, so that we see only the influence of different predictive capacity. For the basic set of parameter values, the $1 \%$ difference leads to a weight of $50.6 \%$ for strategy A while if there were no difference in errors it would be $50 \%$. Hence, a $1 \%$ prediction improvement leads to a $0.6 \%$ switch from predictor B to predictor A.

In Figure 1, inflation rate limit sets are represented over parameter space $(\kappa, \gamma)$ with the basic set of values for other parameters, $\kappa \in(0,0.0002), \gamma \in(0.65,1.25){ }^{3}$ That is, costs vary from non-existent to twice the assumed value, extrapolators predict from $65-125 \%$ of last period's trend will continue. Initial conditions are set at some distance from equilibrium $(0,0.01,0,0.01)$ to avoid seeing only local dynamics.

\section{PLACE FIGURE 1 ABOUT HERE}

$\overline{3}$ All figures are produced with the open-source software iDMC, available (along with model systems used in the paper) at http://code.google.com/p/idmc/. 
The figure (often called a double bifurcation diagram) is essentially a cycle search over parameter space. For every coordinate couple of parameter values the algorithm follows a trajectory and designates with a color (gray scale) the long-run dynamics associated with the given parameter value couple (using the basic set of other parameter values, and from the given initial point). If asymptotic dynamics are a stable fixed point, the coordinate is colored red (gray area on far left). The other colored (gray) areas represent parameter combinations for which stable cycles composed of the indicated number of periodic points exist. The white area represents combinations for which the dynamics are either periodic but of period higher than the maximum cycle sought (12 in the simulations here and in the following), quasiperiodic (and like periodic cycles the sequences lie on an invariant curve), or chaotic (and points lie on a strange attractor).

The black curve superposed on Figure 1 represents the NS bifurcation critical values (13) in Proposition 3.1. The abscissa represents null costs and the equilibrium is stable for values up to the higher bound $\gamma<0.85$, while it is evident that the higher are costs, the closer the critical values come to the lower bound $\gamma=0.675$. That is, the more agents have to pay to form a sophisticated expectation, the smaller is the range for fixed point stability. In the area to the left of the NS curve and to the right of the area of fixed points, the stable $\pi_{n}$ co-exists with other attractors and initial conditions determine to which limit set a trajectory is attracted. This is typical of subcritical NS bifurcations accompanied by a Chenciner bifurcation.

We were not able to derive formally the critical curve of the Chenciner bifurcation (call it $\Gamma$ ), which signals the onset of multiple stable attractors, but conjecture the following. The curve $\Gamma$ intersects the critical curve of the NS bifurcation at $\kappa=0$ suggesting there is no multi-stability for the case of zero cost rational expectations. Then $\Gamma$ slopes to the left following the curve representing loss of fixed point stabil- 
ity until around $\kappa=0.0001$. It is likely that for higher costs $\Gamma$ smoothly converges to the the lower critical bound of $\gamma=0.675$ (more on this point in Section 3.4). In the area of multi-stability between the NS critical curve and $\Gamma$ there is a role for economic policy makers to push the inflation rate from the basin of attraction of a fluctuating limit set into the basin of the equilibrium rate.

Consider also the single bifurcation diagrams (with Lyapunov exponents below) in Figure 2, setting $\kappa=0.0001$. The choice of a predictor making use of rational expectations is insufficient to counterbalance the destabilizing effect of the extrapolative predictor on $\pi_{n}$. Over most of the state space trajectories are attracted to a different limit set, even if $\pi_{n}$ is locally stable until $\gamma_{c} \approx 0.725$. In the next section we describe the dynamics of the inflation rate on attractors in the middle range of the extrapolation coefficient.

\section{PLACE FIGURE 2 ABOUT HERE}

\subsection{Economic dynamics}

The evolution of the first two Lyapunov exponents, plotted in Figure 2 left (bottom), suggest that the attractor type jumps from the equilibrium to a chaotic attractor at around $\gamma=0.7$. A closer look reveals the existence of the period 7 attractor (observable in Figure 1 at $\kappa=0.0001$ ) as well as a tiny interval for quasiperiodic attractors before the interval of strange attractors. In Figure 3 top left, are plots 
of the fixed point, a quasiperiodic attractor and a chaotic attractor $(\gamma=0.675$, $0.7,0.725$, respectively) in the state space $\left(\pi_{t-1}, \pi_{t}\right)$. Figure 3 bottom left plots the fraction of agents using extrapolation $w_{t}^{E}$, which depends on prediction accuracy in the previous period, against the inflation rate $\pi_{t-1}$. For the fixed point, represented in this plot at $\pi_{t-1}=0.3, w_{t}^{E} \approx 78 \%$, both predictors are perfect and RE have costs. The quasiperiodic motion is seen as a torus in the state space (Figure 3 top left), as a U-shaped curve in the $\left(\pi_{t-1}, w_{t}^{E}\right)$ plane (Figure 3 bottom left).

An interval of the time evolution of the inflation rate on the quasiperiodic attractor is represented in Figure 3 top right, the evolution of the trend-following fraction in Figure 3 bottom right. Traces of the nearby period 7 cycle are observable in the inflation rate. We use this quasiperiodic attractor to describe the interaction between macroeconomic mechanisms and the expectations framework because the motion is fairly regular and the influence of periodicity 7 is evident over chaotic attractors as well. Note that the latter implies that there are only a few (3-4) steps from trough to peak (and vice versa). Trend followers obviously have least errors when there is a stable upward or downward movement with slopes corresponding to their extrapolation parameter. Errors of trend-followers increase at the turning point, when the trend stops, and after the turning point, when it turns around.

\section{PLACE FIGURE 3 ABOUT HERE}

From an economic point of view, the model works roughly as follows. Suppose that 
the economy experiences a multi-period increase in the inflation rate and extrapolators are predicting fairly well. Since RE are costly, extrapolators gain in prominence, driving the inflation rate even higher. But inflation rates far from equilibrium trigger macroeconomic mechanisms which tend to dampen deviations. As the inflation rate increases, real money growth declines which, in turn, reduces aggregate demand (via the aggregate demand relation). The reduction in output increases the unemployment rate (via Okun's law). Since this decreases the wage pressure inflation declines (via the Phillips curve). The inflation rate experiences a turn around due to these stabilizing macroeconomic forces and the remaining agents using rational expectations.

After the turning point extrapolators commit significant forecasting errors and, consequently, there is substantial switching to rational expectations. Given their (super) stabilizing impact, the inflation rate then moves toward its normal value. However, during the downward movement of the inflation rate there are still trend-followers who give momentum to the fall and $\pi$ overshoots $\pi_{n}$. A new temporary stabilizing phase is set in motion at the next lower turning around. Some agents switch to extrapolation in the slow down, but back to rational expectations after the turning point. The pattern repeats itself in a quasiperiodic fashion.

In the sample chaotic attractor the interactions are much less regular, although for this nearby (in parameter space) value, trajectories remain close to the invariant curve of the quasiperiodic attractor in the state space (left). Switching is more exaggerated (right) and less regular. For extrapolation coefficients in the zone with intermittency (discussed in detail in Section 3.4), most points are concentrated near $\left(\pi_{n}, w^{E}\left(\pi_{n}\right)\right)$ as trajectories spend more time at the fixed point. For these values of $\gamma$, trend-followers are more accurate predictors and once past turning points, fewer agents switch to rational expectations than for values around the quasiperiodic at- 
tractor. As the extrapolation coefficient is increased the amplitude of fluctuation increases at a slow pace. Even at extreme values, e.g. $\gamma=4$, the inflation rate remains within a finite and economically meaningful interval.

We can now summarize the overall effect of introducing heterogeneity in expectations on the long-run behavior of the model. Recall that in the linear single expectation models, trend-following may lead to a loss of equilibrium inflation rate stability, while rational expectations kept the economy always at $\pi_{n}$. Coupling the macroeconomy to the heterogeneous expectation framework with both predictors leads to the following dynamics.

(i) The super-stabilizing rational expectations predictor is not always able to overcome the destabilizing effect of trend-followers on $\pi_{n}$.

(ii) It is able, in conjunction with the stabilizing forces of the macroeconomy, to overcome the potential global instability of the homogeneous extrapolation model and guarantee the existence of an attractor, albeit typically chaotic, over the relevant range of the extrapolation coefficient.

\subsection{Intermittency}

As observable in Figure 2, the interval for which trend-followers predict the current trend will be amplified $(\gamma>1)$ is characterized by putative chaotic attractors and punctuated by what appear to be returns to fixed point stability. From other simulations it appears that the left limit of the interval moves further left (to values smaller than one) for more reactive switching between predictors (larger $\lambda$ ). A similar observation is evident in the upper right part of Figure 1 for the cost parameter $\kappa$. The odd dynamical behavior of the attractors associated with this seeming return to 
fixed point stability is referred to as intermittency. In the present context, intermittency refers to the fact that the inflation rate is subject to infrequent bouts of large variations in which the trajectory spirals out away from the unstable (saddle-focus) equilibrium until the trajectory suddenly returns to a vicinity of the equilibrium. There it remains for long intervals but, eventually, it will follow a new round of moving away. Economically, this corresponds to a regime where the inflation rate remains quite close to its normal value for an extended period of time but then, with no change in the macroeconomic environment, suddenly becomes more volatile.

These bouts of fluctuations continue for as long as one cares to simulate, a behavior that can be very confusing to the observer. All numerical methods for characterizing attractors depend on the given time interval. Any bifurcation diagram represents the situation after the selected number of transients and over the selected number of iterations. In Figure 1, for example, after 10000 transients the algorithm assigns a fixed point to parameter pairs that are most likely chaotic attractors with intermittency. Lyapunov exponents are time averages and unfortunately do not converge for trajectories with this kind of dynamics (note the exponents' behavior in Figure 2 bottom left, for higher values of $\gamma$ ).

We conclude that if extrapolators believe that the current trend will continue with much the same or even more intensity, there will be long periods of inflation rates near normal, interrupted occasionally by a relatively fast flight of increasing amplitude cycles until the economy returns to near normal inflation rates and the sequence begins anew. Dynamics similar to these were found by Brock and Hommes (1997), in a different context, with rational and naive expectations. 


\subsection{A monetary rule}

It might be argued that the equilibrium point instability introduced by the trendfollowing expectation would be countered by a more flexible monetary rule. As a first attempt to explore this possibility we consider a modified Taylor rule of the following form:

$$
m_{t+1}=m_{n}+a_{\pi}\left(\pi^{*}-\pi_{t}\right)+a_{g}\left(g_{n}-g_{t}\right)
$$

where $m_{n}$ is the money growth target rather than a constant $m$ used in equation (3); $\pi^{*}$ is desired rate of inflation; $a_{\pi}$ and $a_{g}$ are parameters indicating reaction to the current distance of inflation rate from its desired rate and current output growth from its normal rate, respectively. The rule suggests that if inflation is above (below) target or output growth rate is above (below) normal, the relevant authority reacts with a tight (easy) monetary policy.

Our preliminary results are based only on numerical simulations and the following interpretations are to be taken as conjectures. We again set $m_{n}=0.05$ and $\gamma=0.7$, and let $\pi^{*}=\pi_{n}=0.03$. In figure 4 are the double bifurcation diagrams over the monetary authorities reaction parameters, $a_{g}$ against $a_{\pi}$. On the left simulations start from the usual initial conditions at some distance from the equilibrium inflation rate, on the right initial conditions are very near equilibrium. An approximation of the NS bifurcation critical curve is then given by the curve of fixed point stability loss in Figure 4 right. The Chenciner bifurcation critical curve is approximated by the curve of fixed point stability loss in Figure 4 left. The area between these 2 curves is characterized by multi-stability, implying again a role for economic policy. 


\section{PLACE FIGURE 4 ABOUT HERE}

Overall, the output gap reaction part of the Taylor rule seems to be more effective in stabilizing the equilibrium inflation rate - parameter combinations in the upper right part of the bifurcation diagrams in Figure 4 are characterized by fixed point dynamics. If monetary authorities react to the inflation gap they are likely to create a persistent fluctuation in inflation rates. Worse still is setting $a_{\pi}>1$, which leads to a total loss of stability for the system unless $a_{g}$ is simultaneously set high.

This situation contrasts with that of the typical Taylor rule, for which it is often said that one should set $a_{\pi}>1$. But in the original framework an increase in the inflation rate of, say $1 \%$, is accompanied by an increase of the nominal interest rate by more than $1 \%$, implying also a positive increase of the real interest rate which may, in turn, dampen the economy. If $a_{\pi}<1$ the nominal interest rate increases but the real interest rate decreases, and there is no stabilizing effect.

The model studied in this section combines the Taylor rule with a monetary model, and the dynamics are dramatically modified. Here an increase in the inflation rate of $1 \%$ leads to a decrease in money growth for any positive value of $a_{\pi}$. From (3), we have that output decreases because inflation increases and money growth decreases. This double effect means that even if the money growth rate were stable there would be a dampening effect. A further complication is due to the time lag implied by inserting (14) into (3), so that $\pi_{t}$ and $\pi_{t-1}$ affect the dynamics. In this situation causalities may be difficult to reason through.

Nevertheless, if the hypothesis underlying the model are accepted, a judicious 
choice of values for the Taylor rule could prove useful in stabilizing the equilibrium inflation rate. Consider Figure 4 left, with initial conditions some distance from equilibrium. A safe pair of values might be $a_{\pi}=0.25, a_{g}=0.75$. In Figure 2 right we have the same simulations as in Figure 2 left, except for the inclusion of the Taylor rule with the parameter values mentioned above. It is clear that the inflation rate stays stable for a wider range of values of the parameter used by extrapolators.

Of course one must always be cautious about letting implications from abstract models influence policy decisions. The present study suggests that interventions based on simple models of a Taylor rule may result in undesired consequences if there are additional relations between macrovariables and other sources of fluctuations in the real economy. The effectiveness of monetary policy rules in the presence of heterogeneous expectations is also investigated in Anufriev et al. (2008) and De Grauwe (2008).

\section{Concluding remarks}

The heterogeneous expectations framework, for which there is solid empirical support in many economic contexts such as inflation expectations or asset price expectations, naturally opens up the potential dynamics of any model in which it is assumed. The basic ingredients of the framework which lead to long-run fluctuating behavior in the dynamic variables include a destabilizing force, such as extrapolative expectations used by trend followers, a stabilizing force, such as rational expectations and a mechanism for switching between expectations, a weighting of expectations according to some performance criterion. ${ }^{4}$

$\overline{4}$ A study of the model proposed in Section 2 with extrapolative and reversion expectations gives similar results (see early, unpublished version in Lines and Westerhoff (2006b). 
We find that in the case of rational versus extrapolative expectations the model is not destabilized, even by extreme values of the extrapolation coefficient. Rather, much of the relevant parameter space is dominated by chaotic attractors characterized by intermittency, in which trajectories spend much of the time very near the equilibrium inflation rate. Hence, heterogeneous expectation formation is one source of endogenous macroeconomic dynamics. Moreover, a Taylor-like monetary policy rule which gives weight to an output growth gap has some potential in stabilizing the dynamics. This interesting and important research topic clearly needs more attention in the future and we hope that our paper contributes to a fruitful discussion.

\section{Mathematical appendix}

To develop equation (4) we need to determine the factor $\left(\pi_{t}^{e}-\pi_{t-1}^{e}\right)$ in terms of past values of variable $\pi$. We have, from (9) and using the fact that $w_{t}^{E}+w_{t}^{R}=1$

$$
\pi_{t}^{e}=\pi_{t}^{R}+w_{t}^{E}\left(\pi_{t}^{E}-\pi_{t}^{R}\right)
$$

Substituting rational expectations (7) and extrapolative (8) strategies into $(i)$ and rearranging gives

$$
\pi_{t}^{e}=\pi_{t}+w_{t}^{E}\left(-\pi_{t}+(1+\gamma) \pi_{t-1}-\gamma \pi_{t-2}\right)
$$

From equation (10) we can define

$$
\Phi_{t}=a_{t}^{R}-a_{t}^{E}=\left(-\pi_{t-1}+(1+\gamma) \pi_{t-2}-\gamma \pi_{t-3}\right)^{2}-\kappa,
$$

so that, from (11)

$$
w_{t}^{E}=1 /\left(1+e^{\lambda \Phi_{t}\left(\pi_{t-1}, \pi_{t-2}, \pi_{t-3}\right)}\right) .
$$


Next, using $(i)$ and lagging all dynamic variables once, we obtain

$$
\begin{aligned}
\pi_{t}^{e}-\pi_{t-1}^{e}= & \pi_{t}-\pi_{t-1} \\
& +w_{t}^{E}\left(\pi_{t-1}, \pi_{t-2}, \pi_{t-3}\right)\left(-\pi_{t}+(1+\gamma) \pi_{t-1}-\gamma \pi_{t-2}\right) \\
& -w_{t-1}^{E}\left(\pi_{t-2}, \pi_{t-3}, \pi_{t-4}\right)\left(-\pi_{t-1}+(1+\gamma) \pi_{t-2}-\gamma \pi_{t-3}\right) .
\end{aligned}
$$

Substituting in (4) and setting $\Omega=\pi_{t-1}+\pi_{t}^{e}-\pi_{t-1}^{e}$, we can write (12) as

$$
\pi_{t}=\frac{\alpha \beta\left(m-g_{n}\right)}{1+\alpha \beta}+\frac{\Omega\left(\pi_{t-1}, \pi_{t-2}, \pi_{t-3}, \pi_{t-4}\right)}{1+\alpha \beta}
$$

Before expanding $w_{t}^{E}$ and $w_{t-1}^{E}$, consider the dynamics at the fixed point of $(v i)$. We have from (7), (8) and $(i)$ :

$$
\pi^{E}\left(\pi_{n}\right)=\pi^{R}\left(\pi_{n}\right)=\pi_{t}^{e}\left(\pi_{n}\right)=\pi_{t-1}^{e}\left(\pi_{n}\right)=\pi_{n}
$$

which, using (4) and (vi), gives $\pi_{n}=\left(m-g_{n}\right)$. The unique, fixed point inflation rate of the generalized expectations-augmented system is equal to the difference between the growth rates of money and output.

Equation $(v i)$ is a fourth-order difference equation that can be written as a firstorder system employing auxiliary variables $x_{t}=\pi_{t-1}, y_{t}=x_{t-1}=\pi_{t-2}, z_{t}=$ $y_{t-1}=\pi_{t-3}:$

$$
\begin{aligned}
& \pi_{t}=\pi_{t}\left(\pi_{t-1}, x_{t-1}, y_{t-1}, z_{t-1}\right) \\
& x_{t}=\pi_{t-1} \\
& y_{t}=x_{t-1} \\
& z_{t}=y_{t-1} .
\end{aligned}
$$


The Jacobian matrix for system (vii) is

$$
J(\pi)=\left(\begin{array}{cccc}
\frac{\partial \pi_{t}}{\partial \pi_{t-1}} & \frac{\partial \pi_{t}}{\partial x_{t-1}} & \frac{\partial \pi_{t}}{\partial y_{t-1}} & \frac{\partial \pi_{t}}{\partial z_{t-1}} \\
1 & 0 & 0 & 0 \\
0 & 1 & 0 & 0 \\
0 & 0 & 1 & 0
\end{array}\right)
$$

and eigenvalues can be determined explicitly.

At the unique fixed point $\pi_{n}$ we have $\frac{\partial \pi_{t}}{\partial z_{t-1}}=0$ and the characteristic equation for the linear approximation of $(v i)$ using the Jacobian (viii) can be written as

$$
\lambda\left(\lambda^{3}-a \lambda^{2}-b \lambda-c\right)=0 .
$$

The coefficients of $(i x)$ are defined as follows:

$$
\begin{aligned}
& a=\frac{\partial \pi_{t}\left(\pi_{n}\right)}{\partial \pi_{t-1}}=\frac{2 \mu+\gamma \mu}{\alpha \beta+\mu} \\
& b=\frac{\partial \pi_{t}\left(\pi_{n}\right)}{\partial x_{t-1}}=\frac{-2 \mu \gamma-\mu}{\alpha \beta+\mu} \\
& c=\frac{\partial \pi_{t}\left(\pi_{n}\right)}{\partial y_{t-1}}=\frac{\mu \gamma}{\alpha \beta+\mu}
\end{aligned}
$$

setting $\mu=(1+\exp (-\lambda \kappa))^{-1}$.

The potential loss of local stability of the fixed point of system (vii) can be explored by making use of a set of stability conditions for a third order equation (note one eigenvalue is always zero), such as those of Farebrother (1973). The conditions 
used for the polynomial in parentheses in $(i x)$ are

$$
\begin{gathered}
1-a-b-c>0 \\
1+a-b+c>0 \\
1+b+a c-c^{2}>0 \\
3-a+b+3 c>0
\end{gathered}
$$

associated, respectively, with the fold (A), flip (B) and Neimark-Sacker (C) bifurcations. (Proof that no smoothly changing parameter satisfies condition (D) as an equality while simultaneously satisfying conditions (A), (B) and (C) is found in Lines, 2007). It can be shown that (A) and (B) are always satisfied, but that (C) is not. Then the normal inflation rate loses hyperbolicity at $\gamma_{c}=\frac{\alpha \beta+\mu}{2 \mu}$.

\section{References}

Agliari, A., 2006. Homoclinic connections and subcritical Neimark bifurcations in a duopoly model with adaptively adjusted productions. Chaos, Solitons \& Fractals $29,739-755$.

Agliari, A., Bischi, G-I., Gardini, L., 2006. Some methods for the global analysis of business cycle models in discrete time, in: Puu, T., Sushko, I. (Eds.), Business cycle dynamics. Models and Tools, Springer-Verlag, Berlin, pp. 7-47.

Alfarano, S., Lux, T., Wagner, F., 2005. Estimation of agent-based models: The case of an asymmetric herding model. Computational Economics 26, 19-49.

Anufriev, M., Assenza, T., Hommes, C., Massaro, D., 2008. Interest rate rules with heterogeneous expectations. CeNDEF Working Paper 08-08, University of Amsterdam. 
Baak, S.J., 1999. Tests for bounded rationality with a linear dynamic model distorted by heterogeneous expectations. Journal of Economic Dynamics and Control 23, 1517-1543.

Berardi, M., 2007. Heterogeneity and misspecifications in learning. Journal of Economic Dynamics and Control 31, 3203-3227.

Blanchard, O. J., 2005. Macroeconomics, 4th ed. Prentice Hall, New Jersey.

Boswijk, P., Hommes, C., Manzan, S., 2007. Behavioral heterogeneity in stock prices. Journal of Economic Dynamics and Control 31, 1938-1970.

Branch, W., 2004. The theory of rational heterogeneous expectations: evidence from survey data on inflation expectations. Economic Journal 114, 592-621.

Branch, W., 2007. Sticky information and model uncertainty in survey data on inflation expectations. Journal of Economic Dynamics and Control 31, 245-276.

Branch, W., Evans, G., 2006. Intrinsic heterogeneity in expectation formation. Journal of Economic Theory 127, 264-295.

Branch, W., McGough, B., 2006. Dynamic predictor selection in a New Keynesian model with heterogeneous expectations. Working paper.

Branch, W., McGough, B., 2008. A New-Keynesian model with heterogeneous expectations. Journal of Economic Dynamics and Control, forthcoming.

Brock, W., Hommes, C., 1997. A rational route to randomness. Econometrica 65, 1059-1095.

Brock, W., Hommes, C. 1998. Heterogeneous beliefs and routes to chaos in a simple asset pricing model. Journal of Economic Dynamics Control 22, 1235-1274.

Carrol, C., 2003. Macroeconomic expectations of households and professional forecasters. Quarterly Journal of Economics 118, 269-298.

Chavas, J.P., 2000. On information and market dynamics: the case of the U.S. beef market. Journal of Economic Dynamics and Control 24, 833-853.

Chiarella, C., Dieci, R., Gardini, L., 2002. Speculative behaviour and complex asset 
price dynamics: A global analysis. Journal of Economic Behavior and Organization 49, 173-197.

Day, R., 1999. Complex Economic Dynamics: An Introduction to Macroeconomic Dynamics, Vol. 2. MIT Press, Cambridge.

Day, R., Huang, W., 1990. Bulls, bears and market sheep. Journal of Economic Behavior and Organization 14, 299-329.

De Grauwe, P., 2008. DSGE-modelling when agents are imperfectly informed. ECB Working Paper 897, European Central Bank.

Evans, G., Honkapohja, S., 2001. Learning and expectations in macroeconomics. Princeton University Press, Princeton.

Farebrother, R., 1973. Simplified Samuelson conditions for cubic and quartic equations. The Manchester School of Economics and Social Studies 41, 396-400.

Franke, R., 2007. A sophisticatedly simple alternative to the New-Keynesian Phillips curve, in: Asada, T., Ishikawa, T. (Eds.), Time and Space in Economics. Springer, Berlin, pp. 3-28.

Gaunersdorfer, A., Hommes, C., Wagener, F., 2008. Bifurcation routes to volatility clustering under evolutionary learning. Journal of Economic Behavior and Organization 67, 27-47.

Heemeijer, P., Hommes, C., Sonnemans, J., Tuinstra, J., 2009. Price stability and volatility in markets with positive and negative expectations feedback: an experimental investigation, Journal of Economic Dynamics and Control 33, 1052-1072. Hommes, C., Sonnemans, J., Tuinstra, J., van de Velden, H., 2005. Coordination of expectations in asset pricing experiments. Review of Financial Studies 18, 955-980.

Hommes, C., Sonnemans, J., Tuinstra, J., van de Velden, H., 2008. Expectations and bubbles in asset pricing experiments. Journal of Economic Behavior and Organization 67, 116-133. 
Honkapohja, S., Mitra, K., 2006. Learning stability in economies with heterogeneous agents. Review of Economic Dynamics 9, 284-309.

Kahneman, D., Slovic, P., Tversky, A., 1986. Judgment under Uncertainty: Heuristics and Biases. Cambridge University Press, Cambridge.

Lines, M. (Ed.), 2005. Nonlinear Dynamical Systems in Economics. Springer, New York.

Lines, M., 2007a. Bifurcation scenarios in a heterogeneous agent, multiplieraccelerator model. Pure Mathematics and Applications 16, 429-442.

Lines, M., 2007b. Practical tools for identifying dynamics in discrete systems. Working paper 3.2007 Department of Statistics, University of Udine.

Lines, M., Westerhoff, F., 2006a. Expectations and the multiplier-accelerator model, in: Puu, T., Sushko, I. (Eds.), Business Cycle Dynamics. Models and Tools, Springer-Verlag, Berlin, pp. 255-276.

Lines, M., Westerhoff, F., 2006b. Expectation formation and macroeconomic dynamics. Working paper 13.2006 Department of Statistics, University of Udine.

Lux, T., 1995. Herd behavior, bubbles and crashes. Economic Journal 105, 881896.

Mankiw, N.G., Reis, R., Wolfers, J., 2003. Disagreement about inflation expectations. NBER Macroeconomic Annual 18, 209-248.

Manski, C.F., McFadden, D., 1981. Structural Analysis of Discrete Data with Econometric Applications. MIT Press, Cambridge.

Marcet, A., Nicolini, J., 2003. Recurrent hyperinflations and learning. American Economic Review 93, 1476-1498.

Medio, A., 1992. Chaotic Dynamics. Cambridge University Press, Cambridge.

Medio, A., Lines, M., 2001. Nonlinear Dynamics: a Primer. Cambridge University Press, Cambridge.

Pesaran, H., Weale, M., 2006. Survey expectations, in: Granger, C., Timmerman, 
A. (Eds.), Handbook of Economic Forecasting, North-Holland, Amsterdam, pp. 715-776.

Puu, T., Sushko, I. (Eds.), 2006. Business Cycle Dynamics: Models and Tools. Springer-Verlag, Berlin.

Rosser, J. B., 2000. From Catastrophe to Chaos: A General Theory of Economic Discontinuities, 2nd ed. Kluwer Academic Publishers, Boston.

Simon, H. A., 1955. A behavioral model of rational choice. Quarterly Journal of Economics 9, 99-118.

Smith, V., 1991. Papers in Experimental Economics. Cambridge University Press, Cambridge.

Tuinstra, J., Wagener, F., 2007. On learning equilibria. Economic Theory 30,493513.

Westerhoff, F., 2006a. Samuelson's multiplier-accelerator model revisited. Applied Economics Letters 13, 89-92.

Westerhoff, F., 2006b. Business cycles, heuristic expectation formation and contracyclical policies. Journal of Public Economic Theory 8, 821-838.

Westerhoff, F., Reitz, S., 2003. Nonlinearities and cyclical behavior: the role of chartists and fundamentalists. Studies in Nonlinear Dynamics and Econometrics 7, no. 4, article 3 . 


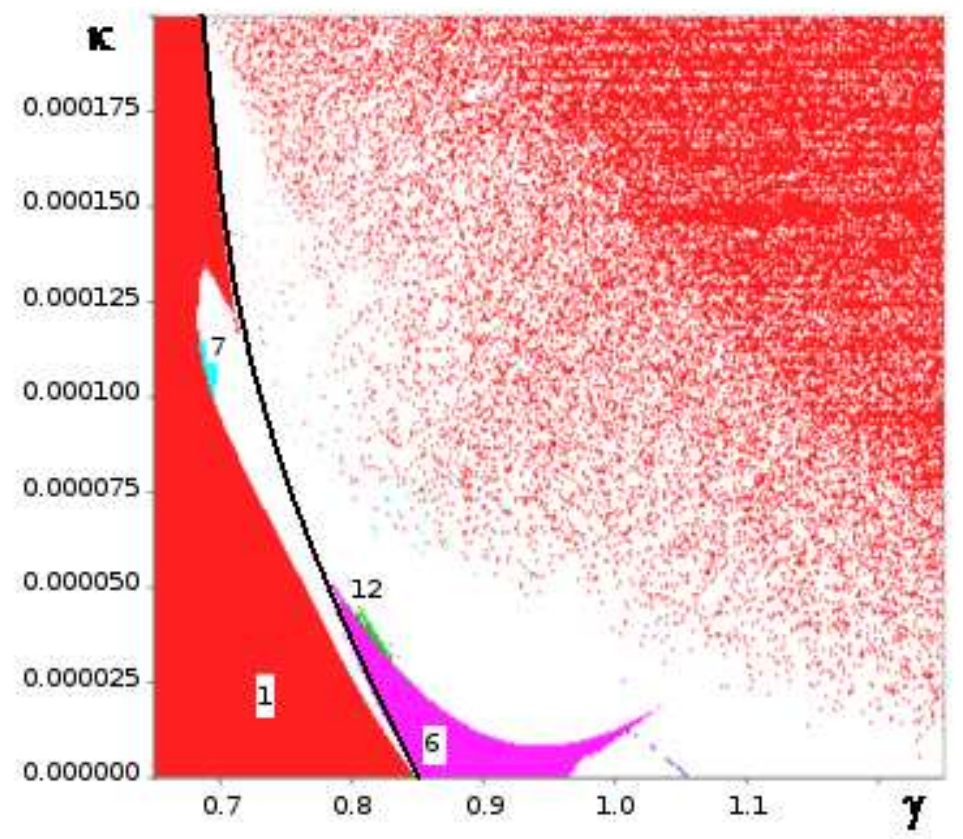

Fig. 1. Limit sets in parameter space $(\gamma, \kappa)$. 

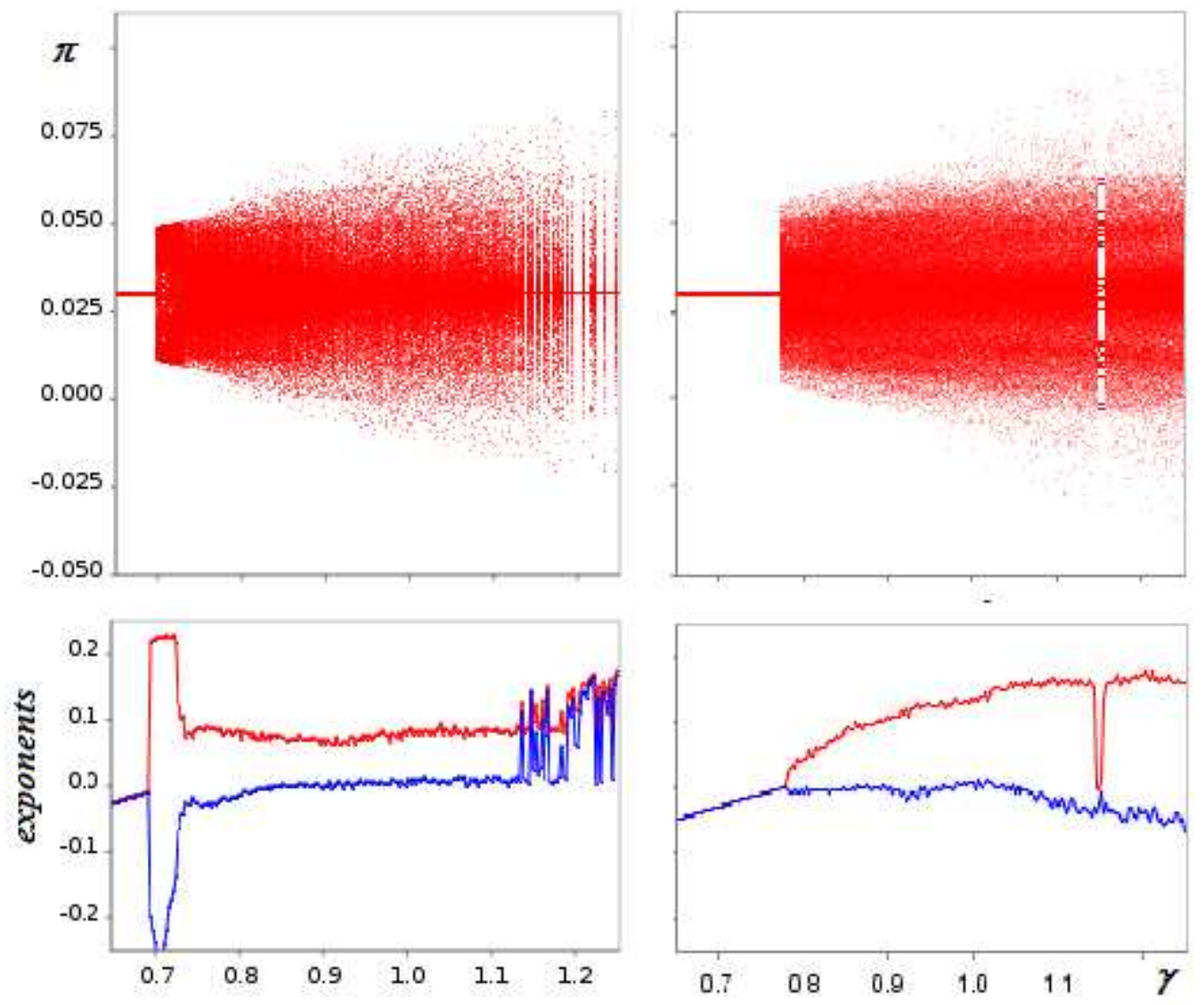

Fig. 2. Top: bifurcation diagrams; bottom: LCEs. Left without, right with monetary rule 

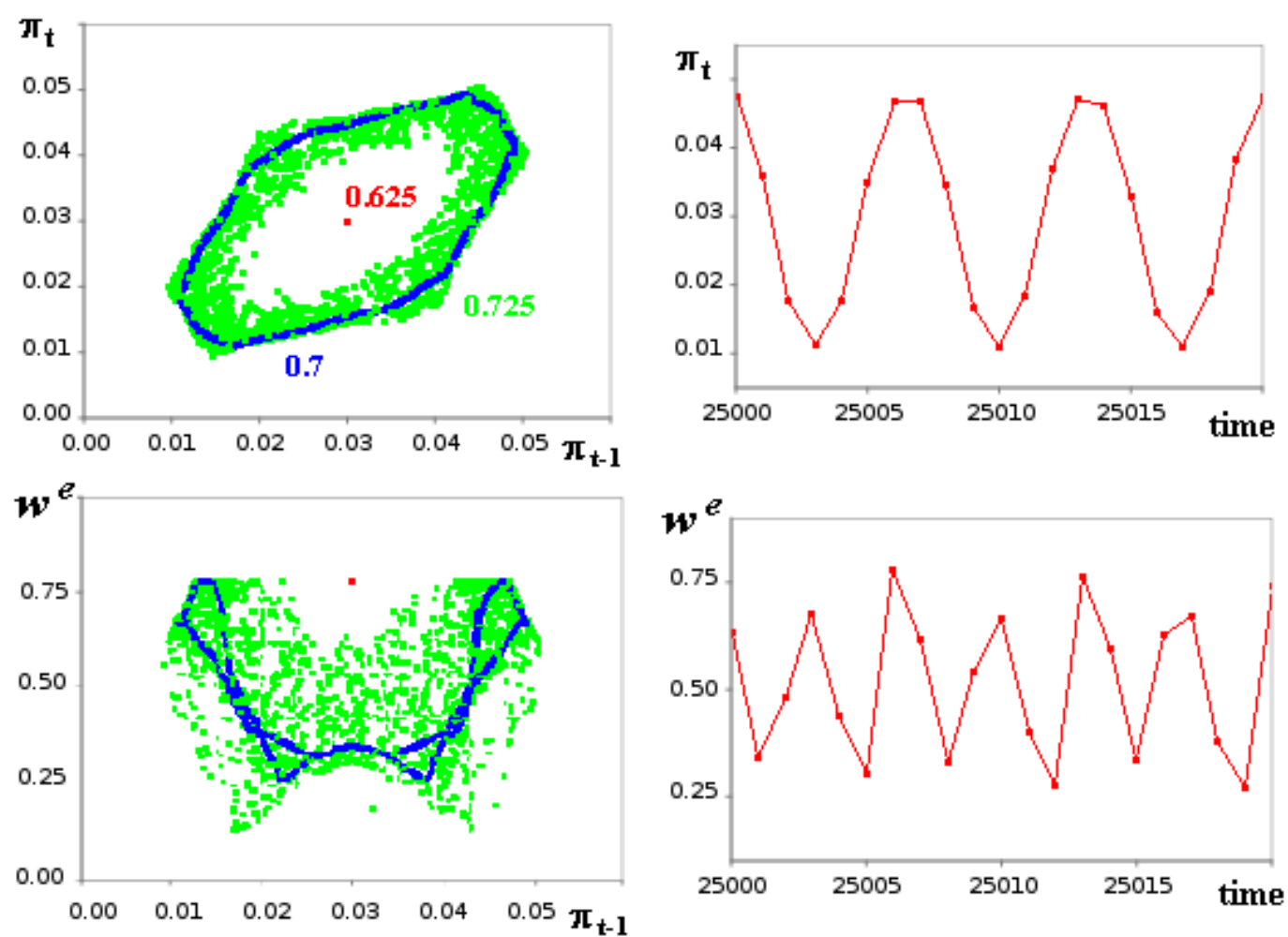

Fig. 3. Top left: state space $\left(\pi_{t-1}, \pi_{t}\right)$; bottom left: $\left(\pi_{t-1}, w_{t}^{e}\right)$. Top right: time slice of $\pi_{t}$; bottom right: time slice of $w_{t}^{e}$.
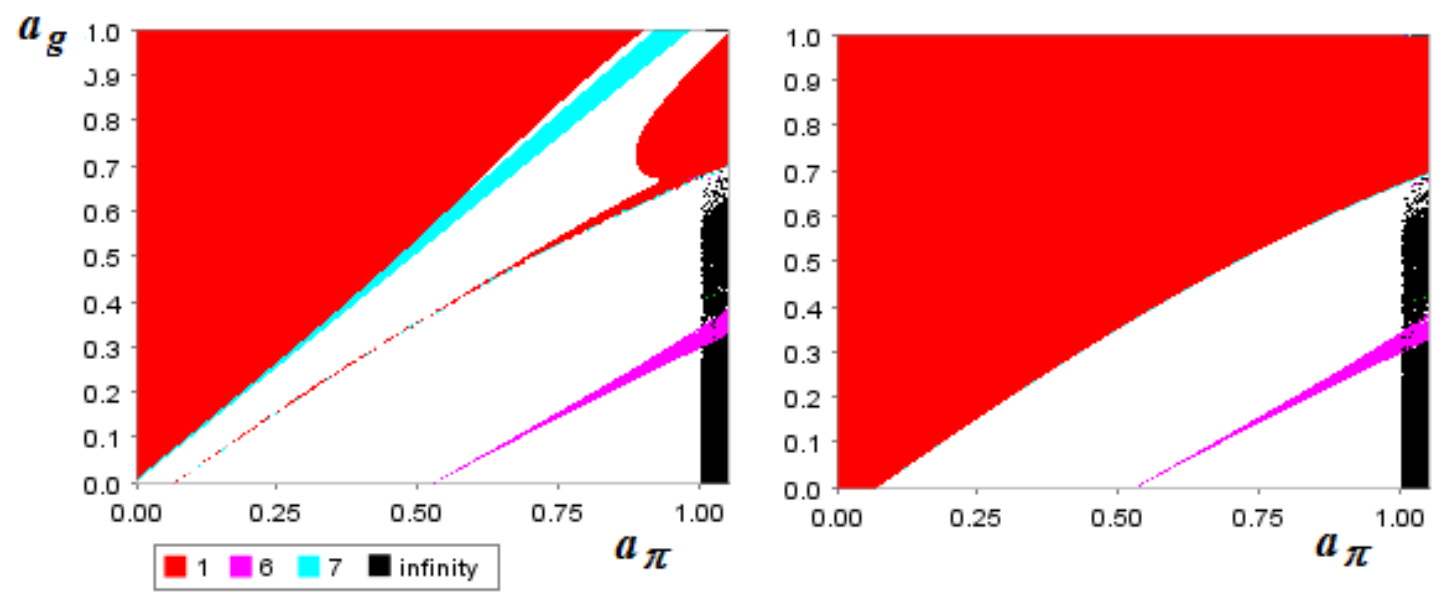

Fig. 4. Limit sets over Taylor coefficients. Left distant, right near equilibrium initial values. 